\title{
A STUDY OF SOME NEW ABSOLUTE SUMMABILITY METHODS
}

\author{
W.T. SULAIMAN \\ P O Box 11985 \\ Sana'a, YEMEN
}

(Received March 30, 1993 and in revised form September 6, 1996)

\begin{abstract}
In this note we introduce a new method of absolute summability. A general theorem is
\end{abstract} given. Several results are also deduced.

KEY WORDS AND PHRASES. Summability.

1991 AMS SUBJECT CLASSIFICATION CODES. Primary 40G99.

\section{INTRODUCTION.}

Let $\sum a_{n}$ be an infinite series with partial sums $s_{n}$. Let $\sigma_{n}^{\delta}$ and $\eta_{n}^{\delta}$ denotes the nth Cesaro mean of order $\delta(\delta>-1)$ of the sequences $\left\{s_{n}\right\}$ and $\left\{n a_{n}\right\}$ respectively. The series $\sum a_{n}$ is said to be summable $|C, \delta|_{k}, k \geq 1$, if

$$
\sum_{n=1}^{\infty} n^{k-1}\left|\sigma_{n}^{\delta}-\sigma_{n-1}^{\delta}\right|^{k}<\infty
$$

or equivalently

$$
\sum_{n=1}^{\infty} n^{-1}\left|\eta_{n}^{\delta}\right|^{k}<\infty
$$

Let $\left\{p_{n}\right\}$ be a sequence of real or complex constants with

$$
P_{n}=p_{0}+p_{1}+\ldots+p_{n}, \quad P_{-1}=p_{-1}=0 .
$$

The series $\sum a_{n}$ is said to be summable $\left|N, p_{n}\right|$, if

$$
\sum_{n=1}^{\infty}\left|t_{n}-t_{n-1}\right|<\infty
$$

where

$$
t_{n}=P_{n}^{-1} \sum_{v=0}^{n} p_{n-v} s_{v}\left(t_{-1}=0\right)
$$

We write $p=\left\{p_{n}\right\}$ and

$$
M=\left\{p: p_{n}>0 \& p_{n+1} / p_{n} \leq p_{n+2} / p_{n+1}, n=0,1, \ldots\right\}
$$

It is known that for $p \in M$, (1) holds if and only if (Das [4])

$$
\sum_{n=1}^{\infty} \frac{1}{n P_{n}}\left|\sum_{v=1}^{n} p_{n-v} v a_{v}\right|<\infty .
$$


DEFINITION 1 (Sulaiman [5]). For $p \in M$, we say that $\sum a_{n}$ is summable $\left|N, p_{n}\right|_{k}, k \geq 1$, if

$$
\sum_{n=1}^{\infty} \frac{1}{n P_{n}^{k}}\left|\sum_{v=1}^{n} p_{n-v} v a_{v}\right|^{k}<\infty
$$

In the special case in which $p_{n}=A_{n}^{r-1}, r>-1$, where $A_{n}^{r}$ is the coefficient of $x^{n}$ in the power series expansion of $(1-x)^{-r-1}$ for $|x|<1,\left|N, p_{n}\right| k$ summability reduces to $|C, r|_{k}$ summability.

The series $\sum a_{n}$ is said to be summable $\left|R, p_{n}\right| k,\left|\bar{N}, p_{n}\right|_{k}, k \geq 1$ (Bor [2] \& [1]), if

$$
\sum_{n=1}^{\infty} n^{k-1}\left|T_{n}-T_{n-1}\right|^{k}<\infty, \sum_{n=1}^{\infty}\left(\frac{P_{n}}{p_{n}}\right)^{k-1}\left|T_{n}-T_{n-1}\right|^{k}<\infty,
$$

respectively, where

$$
T_{n}=P_{n}^{-1} \sum_{v=0}^{n} p_{v} s_{v}
$$

In the special case when $p_{n}=1$ for all values of $n$ (resp. $k=1$ ), then $\left|R, p_{n}\right|_{k},\left|\bar{N}, p_{n}\right|_{k}$ summability is the same as $|C, 1|_{k}$ (resp. $\left.\left|R, p_{n}\right|\right)$ summability.

We set

$$
\begin{aligned}
Q_{n} & =q_{0}+q_{1}+\ldots+q_{n}, \quad q_{-1}=Q_{-1}=0 . \\
U_{n} & =u_{0}+u_{1}+\ldots+u_{n}, \quad u_{-1}=U_{-1}=0 . \\
R_{n} & =p_{0} q_{n}+p_{1} q_{n-1}+\ldots p_{n} q_{0} \\
\triangle f_{n} & =f_{n}-f_{n+1}
\end{aligned}
$$

We assume $\left\{\phi_{n}\right\},\left\{\alpha_{n}\right\}$ and $\left\{\beta_{n}\right\}$ be sequences of positive real constants. Here we give the following new definition.

DEFINITION 2. Let $\left\{p_{n}\right\},\left\{q_{n}\right\}$ be sequences of positive real constants such that $q \in M$ We say that $\sum a_{n}$ is summable $\left|N, R_{n}, \phi_{n}\right|_{k}, k \geq 1$, if

$$
\sum_{n=1}^{\infty} \phi_{n}^{k-1}\left|\frac{p_{n}}{P_{n} R_{n-1}} \sum_{v=1}^{n} P_{v-1} q_{n-v} a_{v}\right|^{k}<\infty
$$

DEFINITION 3 (Sulaiman [6]). The series $\sum a_{n}$ is said to be summable $\left|\bar{N}, p_{n}, \phi_{n}\right|_{k}, k \geq 1$, if

$$
\sum_{n=1}^{\infty} \phi_{n}^{k-1}\left|T_{n}-T_{n-1}\right|^{k}<\infty
$$

\section{LEMMAS}

LEMMA 1. Let $\left\{p_{n}\right\},\left\{q_{n}\right\}$, and $\left\{u_{n}\right\}$ be sequences of positive real constants such that $q \in M$, $\left\{\alpha_{n}^{1-1 / k} p_{n} / P_{n} R_{n-1}\right\}$ nonincreasing for $q_{n} \neq c$. Let $T_{n}$ denote the $\left(\bar{N}, u_{n}\right)$-mean of the series $\sum a_{n}$ Let $\left[\epsilon_{n}\right\}$ be a sequence of constants and write $\beta_{n}^{1-1 / k} \Delta T_{n-1}=\Delta_{n}$ If

$$
\begin{gathered}
\sum_{n=v+1}^{m+1} \frac{\alpha_{n}^{k-1} p_{n}^{k}}{P_{n}^{k} R_{n-1}} q_{n-v-1}=0\left\{\frac{\alpha_{v}^{k-1} p_{v}^{k-1}}{P_{v}^{k}}\right\}, \\
\sum_{n=1}^{\infty}\left(\frac{\alpha_{n}}{\beta_{n}}\right)^{k}\left(\frac{p_{n}}{P_{n}}\right)^{k}\left(\frac{P_{n-1}}{R_{n-1}}\right)^{k}\left(\frac{U_{n}}{u_{n}}\right)^{k}\left|\epsilon_{n}\right|^{k}\left|\triangle_{n}\right|^{k}<\infty, \\
\sum_{n=1}^{\infty}\left(\frac{\alpha_{n}}{\beta_{n}}\right)^{k-1}\left|\epsilon_{n}\right|^{k}\left|\triangle_{n}\right|^{k}<\infty,
\end{gathered}
$$


and

$$
\sum_{n=1}^{\infty}\left(\frac{\alpha_{n}}{\beta_{n}}\right)^{k-1}\left(\frac{U_{n-1}}{u_{n}}\right)^{k}\left|\Delta \epsilon_{n}\right|^{k}\left|\Delta_{n}\right|^{k}<\infty
$$

then the series $\sum a_{n} \epsilon_{n}$ is summable $\left|N, R_{n}, \alpha_{n}\right|_{k}, k \geq 1$.

LEMMA 2 (Sulaiman [7]) Let $q \in M$. Then for $0<r \leq 1$,

$$
\sum_{n=v+1}^{\infty} \frac{q_{n-v-1}}{n^{r} Q_{n-1}}=0\left(v^{-r}\right) \text {. }
$$

LEMMA 3 (Bor [2]). Let $k>1$ and $A=\left(a_{n v}\right)$ be an infinite matrix. In order that $A \in\left(l^{k} ; l^{k}\right)$, it is necessary that

$$
a_{n v}=0(1) \quad(\text { all } n, v)
$$

Proof of Lemma 1. Write

$$
\tau_{n}=\sum_{v=1}^{n} P_{v-1} q_{n-v} a_{v} \epsilon_{v}
$$

Since

$$
T_{n}=U_{n}^{-1} \sum_{v=0}^{n} u_{v} \sum_{r=0}^{v} a_{r}=U_{n}^{-1} \sum_{v=0}^{n}\left(U_{n}-U_{v-1}\right) a_{v}
$$

then

$$
-\Delta T_{n-1}=\frac{u_{n}}{U_{n} U_{n-1}} \sum_{v=1}^{n} U_{v-1} a_{v}
$$

By Abel's transformation,

$$
\begin{aligned}
& \tau_{n}=\sum_{v=1}^{n} U_{v-1} a_{v}\left(P_{v-1} q_{n-v} U_{v-1}^{-1} \epsilon_{v}\right) \\
& =\sum_{v=1}^{n-1}\left(\sum_{r=1}^{v} U_{r-1} a_{r}\right) \Delta\left(P_{v-1} q_{n-v} U_{v-1}^{-1} \epsilon_{v}\right)+\left(\sum_{r=1}^{n} U_{r-1} a_{r}\right) P_{n-1} q_{0} U_{n-1}^{-1} \epsilon_{n} \\
& =\sum_{v=1}^{n-1}\left\{-\frac{U_{v-1} U_{v}}{u_{v}} \Delta T_{v-1}\right\}\left\{P_{v-1} \Delta_{v} q_{n-v} U_{v-1}^{-1} \epsilon_{v}+P_{v-1} q_{n-v-1} \frac{u_{v}}{U_{v-1} U_{v}} \epsilon_{v}\right. \\
& \left.-p_{v} q_{n-v-1} U_{v}^{-1} \epsilon_{v}+P_{v} q_{n-v-1} U_{v}^{-1} \Delta \epsilon_{v}\right\}-P_{n-1} q_{0} U_{n} u_{n}^{-1} \epsilon_{n} \Delta T_{n-1} \\
& =\sum_{v=1}^{n-1}\left\{-P_{v-1} \Delta q_{n-v} \frac{U_{v}}{u_{v}} \epsilon_{v} \Delta T_{v-1}-P_{v-1} q_{n-v-1} \epsilon_{v} \Delta T_{v-1}+p_{v} q_{n-v-1} \frac{U_{v-1}}{u_{v}}\right. \\
& \left.\times \epsilon_{v} \Delta T_{v-1}-P_{v} q_{n-v-1} \frac{U_{v-1}}{u_{v}} \Delta \epsilon_{v} \Delta T_{v-1}\right\}-P_{n-1} q_{0} \frac{U_{n}}{u_{n}} \epsilon_{n} \Delta T_{n-1} \\
& =\tau_{n, 1}+\tau_{n, 2}+\tau_{n, 3}+\tau_{n, 4}+\tau_{n, 5} \text {, say }
\end{aligned}
$$

In order to prove the lemma, by Minkowski's inequality, it is sufficient to show that

$$
\sum_{n=1}^{\infty} \alpha_{n}^{k-1}\left|\frac{p_{n}}{P_{n} R_{n-1}} \tau_{n, r}\right|^{k}<\infty, \quad r=1,2,3,4,5
$$

Applying Hölder's inequality, 


$$
\begin{aligned}
& \sum_{m=2}^{m+1} \alpha_{n}^{k-1}\left|\frac{p_{n}}{P_{n} R_{n-1}} \tau_{n, 1}\right|^{k}=\sum_{n=2}^{m+1} \alpha_{n}^{k-1}\left|\frac{p_{n}}{P_{n} R_{n-1}} \sum_{v=1}^{n-1} P_{v-1} \triangle_{v} q_{n-v} \frac{U_{v}}{u_{v}} \epsilon_{v} \Delta T_{v-1}\right|^{k} \\
& \leq \sum_{n=2}^{m+1} \alpha_{n}^{k-1}\left(\frac{p_{n}}{P_{n} R_{n-1}}\right)^{k} \sum_{v=1}^{n-1} P_{v-1}^{k}\left|\triangle_{v} q_{n-v}\right|\left(\frac{U_{v}}{u_{v}}\right)^{k}\left|\epsilon_{v}\right|^{k}\left|\triangle T_{v-1}\right|^{k} \\
& \times\left\{\sum_{v=1}^{n-1}\left|\triangle_{v} q_{n-v}\right|\right\}^{k-1} \\
& =0(1) \sum_{v=1}^{m} P_{v-1}^{k}\left(\frac{U_{v}}{u_{v}}\right)^{k}\left|\epsilon_{v}\right|^{k}\left|\triangle T_{v-1}\right|^{k} \sum_{n=v+1}^{m+1} \frac{\alpha_{n}^{k-1} p_{n}^{k}}{P_{n}^{k} R_{n-1}^{k}}\left|\triangle_{v} q_{n-v}\right| \\
& =0(1) \sum_{v=1}^{m}\left(\frac{\alpha_{v}}{\beta_{v}}\right)^{k-1}\left(\frac{p_{v}}{P_{v}}\right)^{k}\left(\frac{P_{v-1}}{R_{v-1}}\right)^{k}\left(\frac{U_{v}}{u_{v}}\right)^{k}\left|\epsilon_{v}\right|^{k}\left|\triangle_{v}\right|^{k} \\
& \begin{aligned}
\sum_{n=2}^{m+1} \alpha_{n}^{k-1}\left|\frac{p_{n}}{P_{n} R_{n-1}} \tau_{n, 2}\right|^{k} & =\sum_{n=2}^{m+1} \alpha_{n}^{k-1}\left|\frac{p_{n}}{P_{n} R_{n-1}} \sum_{v=1}^{n-1} \frac{P_{v-1}}{p_{v}} p_{v} q_{n-v-1} \epsilon_{v} \Delta T_{v-1}\right|^{k} \\
& \leq \sum_{n=2}^{m+1} \frac{\alpha_{n}^{k-1} p_{n}^{k}}{P_{n}^{k} R_{n-1}} \sum_{v=1}^{n-1}\left(\frac{P_{v-1}}{p_{v}}\right)^{k} p_{v} q_{n-v-1}\left|\epsilon_{v}\right|^{k}\left|\Delta T_{v-1}\right|^{k}\left\{\sum_{v=1}^{n-1} \frac{p_{v} q_{n-v-1}}{R_{n-1}}\right\}^{k-1} \\
& =0(1) \sum_{v=1}^{m}\left(\frac{P_{v}}{p_{v}}\right)^{k} p_{v}\left|\epsilon_{v}\right|^{k}\left|\triangle T_{v-1}\right|^{k} \sum_{n=v+1}^{m+1} \frac{\alpha_{n}^{k-1} p_{n}^{k}}{P_{n}^{k} R_{n-1}} q_{n-v-1} \\
& =0(1) \sum_{v=1}^{m}\left(\frac{\alpha_{v}}{\beta_{v}}\right)^{k-1}\left|\epsilon_{v}\right|^{k}\left|\triangle_{v}\right|^{k}
\end{aligned} \\
& \sum_{m=2}^{m+1} \alpha_{n}^{k-1}\left|\frac{p_{n}}{P_{n} R_{n-1}} \tau_{n, 3}\right|^{k}=\sum_{n=2}^{m+1} \alpha_{n}^{k-1}\left|\frac{p_{n}}{P_{n} R_{n-1}} \sum_{v=1}^{n-1} p_{v} q_{n-v-1} \frac{U_{v-1}}{u_{v}} v \epsilon_{v} \Delta T_{v-1}\right|^{k} \\
& \leq \sum_{n=2}^{m+1} \frac{\alpha_{n}^{k-1} p_{n}^{k}}{P_{n}^{k} R_{n-1}} \sum_{v=1}^{n-1} p_{v} q_{n-v-1}\left(\frac{U_{v-1}}{u_{v}}\right)^{k}\left|\epsilon_{v}\right|^{k}\left|\Delta T_{v-1}\right|^{k} \\
& \times\left\{\sum_{v=1}^{n-1} \frac{p_{v} q_{n-v-1}}{R_{n-1}}\right\}^{k-1} \\
& =0(1) \sum_{v=1}^{m} p_{v}\left(\frac{U_{v}}{u_{v}}\right)^{k}\left|\epsilon_{v}\right|^{k}\left|\Delta T_{v-1}\right|^{k} \sum_{n=v+1}^{m+1} \frac{\alpha_{n}^{k-1} p_{n}^{k}}{P_{n}^{k} R_{n-1}^{k}} q_{n-v-1} \\
& =0(1) \sum_{v=1}^{m}\left(\frac{\alpha_{v}^{k-1}}{\beta_{v}}\right)\left(\frac{p_{v}}{P_{v}}\right)^{k}\left(\frac{P_{v-1}}{R_{v-1}}\right)^{k}\left(\frac{U_{v}}{u_{v}}\right)^{k}\left|\epsilon_{v}\right|^{k}\left|\triangle_{v}\right|^{k}
\end{aligned}
$$




$$
\begin{aligned}
& \sum_{m=2}^{m+1} \alpha_{n}^{k-1}\left|\frac{p_{n}}{P_{n} R_{n-1}} \tau_{n, 4}\right|^{k}=\sum_{n=2}^{m+1} \alpha_{n}^{k-1}\left|\frac{p_{n}}{P_{n} R_{n-1}} \sum_{v=1}^{n-1} P_{v} q_{n-v-1} \frac{U_{v-1}}{u_{v}} \Delta \epsilon_{v} \Delta T_{v-1}\right|^{k} \\
& \leq \sum_{n=2}^{m+1} \frac{\alpha_{n}^{k-1} p_{n}^{k}}{P_{n}^{k} R_{n-1}} \sum_{v=1}^{n-1}\left(\frac{P_{v}}{p_{v}}\right)^{k} p_{v} q_{n-v}\left(\frac{U_{v-1}}{u_{v}}\right)^{k}\left|\Delta \epsilon_{v}\right|^{k}\left|\Delta T_{v-1}\right|^{k} \\
& \times\left\{\sum_{v=1}^{n-1} \frac{p_{v} q_{n-v-1}}{R_{n-1}}\right\}^{k-1} \\
& =0(1) \sum_{v=1}^{m}\left(\frac{P_{v}}{p_{v}}\right)^{k} p_{v}\left(\frac{U_{v-1}}{u_{v}}\right)^{k}\left|\Delta \epsilon_{v}\right|^{k}\left|\Delta T_{v-1}\right|^{k} \cdot \sum_{n=v+1}^{m+1} \frac{\alpha_{n}^{k-1} p_{n}}{P_{n}^{k} R_{n-1}} q_{n-v-1} \\
& =0(1) \sum_{v=1}^{m}\left(\frac{\alpha_{v}}{\beta_{v}}\right)^{k-1}\left(\frac{\alpha_{v}^{k-1}}{\beta_{v}}\right)\left(\frac{U_{v}}{u_{v}}\right)^{k}\left|\Delta \epsilon_{v}\right|^{k}\left|\triangle_{v}\right|^{k} \\
& \sum_{n=1}^{m} \alpha_{n}^{k-1}\left|\frac{p_{n}}{P_{n} R_{n-1}} \tau_{n, 5}\right|^{k}=\sum_{n=1}^{m} \alpha_{n}^{k-1}\left|\frac{p_{n}}{P_{n R_{n-1}}} P_{n-1} q_{0} \frac{U_{n}}{u_{n}} \epsilon_{n} \Delta T_{n-1}\right|^{k} \\
& =0(1) \sum_{n=1}^{m}\left(\frac{\alpha_{n}}{\beta_{n}}\right)^{k-1}\left(\frac{p_{n}}{P_{n}}\right)^{k}\left(\frac{P_{n-1}}{R_{n-1}}\right)^{k}\left(\frac{U_{n}}{u_{n}}\right)^{k}\left|\epsilon_{n}\right|^{k}\left|\triangle_{n}\right|^{k} \text {. }
\end{aligned}
$$

This completes the proof of Lemma 1.

\section{MAIN RESULT}

THEOREM. Let $q \in M$ such that $\left\{\alpha_{n}^{1-1 / k} p_{n} / P_{n} R_{n-1}\right\}$ nonincreasing for $q_{n} \neq c$. Let $P_{n} R_{n-1} u_{n}=0\left(p_{n} P_{n-1} U_{n}\right), \sum \alpha_{n}^{k-1}\left(p_{n} / P_{n}\right)^{k}$ divergent, and

$$
\sum_{n=v}^{\infty} \frac{\alpha_{n}^{k-1} p_{n}^{k}}{P_{n}^{k} R_{n-1}} q_{n-v-1}=0\left\{\frac{\alpha_{v}^{k-1} p_{v}^{k-1}}{P_{v}^{k}}\right\}
$$

Then the necessary and sufficient conditions that $\sum a_{n} \epsilon_{n}$ is summable $\left|N, R_{n}, \alpha_{n}\right|_{k}$ whenever $\sum a_{n}$ is summable $\left|\bar{N}, p_{n}, \beta_{n}\right|_{k}, k \geq 1$, are

$$
\text { (i) } \begin{aligned}
\epsilon_{n} & =0\left\{\left(\frac{P_{n} R_{n-1} u_{n}}{p_{n} P_{n-1} U_{n}}\right)\left(\frac{\beta_{n}}{\alpha_{n}}\right)^{1-1 / k}\right. \\
\text { (ii) } \quad \Delta \epsilon_{n} & =0\left\{\left(\frac{u_{n}}{U_{n-1}}\right)\left(\frac{\beta_{n}}{\alpha_{n}}\right)^{1-1 / k}\right\} .
\end{aligned}
$$

PROOF. Sufficiency. Follows form Lemma 1.

Necessity of (i). Multiplying (2) by $\alpha_{n}^{1-1 / k} p_{n} / P_{n} R_{n-1}$, the last term on the right becomes

$$
\begin{aligned}
\frac{\alpha_{n}^{1-1 / k} p_{n}}{P_{n} R_{n-1}} \tau_{n, 5} & =-\frac{p_{n} P_{n-1} U_{n}}{P_{n} R_{n-1} u_{n}} \epsilon_{n} \Delta T_{n-1} \\
& =-\left\{\frac{p_{n} P_{n-1} U_{n}}{P_{n} R_{n-1} u_{n}}\left(\frac{\alpha_{n}}{\beta_{n}}\right)^{1-1 / k} \epsilon_{n}\right\}\left(\beta_{n}^{1-1 / k} \Delta T_{n-1}\right)
\end{aligned}
$$

Following Bor [2]. By (3), it is possible to write the matrix transforming $\left(\beta_{n}^{1-1 / k} \Delta T_{n-1}\right)$ into $\left(\left(\alpha_{n}^{1-1 / k} p_{n} / P_{n} R_{n-1}\right) \tau_{n}\right)$. Since $\left|\bar{N}, p_{n}, \beta_{n}\right|_{k}$ implies $\left|N, R_{n}, \alpha_{n}\right|_{k}$, the matrix $\epsilon\left(l^{k} ; l^{k}\right)$. By Lemma 3, a necessary condition for this implication is that the elements (in particular the diagonal elements) of this matrix should be bounded. Hence (i) 
Necessity of (ii). Suppose $\left|\bar{N}, p_{n}, \beta_{n}\right|_{k}$ of $\sum a_{n}$ implies $\left|N, R_{n}, \alpha_{n}\right|_{k}$ of $\sum a_{n} \epsilon_{n}$. From (2)

$$
\left|\tau_{n, 4}\right| \leq \sum_{r}\left|\tau_{n, r}\right|+\left|\tau_{n}\right|, \quad r=1,2,3,5
$$

By Minkowski's inequality, using (i), we have, via the proof of Lemma 1,

$$
\begin{aligned}
\sum_{n=1}^{m} \alpha_{n}^{k-1}\left(\frac{p_{n}}{P_{n} R_{n-1}}\right)^{k}\left|\tau_{n, 4}\right|^{k} & \leq 0(1)\left\{\sum_{n=1}^{m} \alpha_{n}^{k-1}\left(\frac{p_{n}}{P_{n} R_{n-1}}\right)^{k}\left|\tau_{n, r}\right|^{k}+\sum_{n=1}^{m} \alpha_{n}^{k-1}\left(\frac{p_{n}}{P_{n} R_{n-1}}\right)^{k}\left|\tau_{n}\right|^{k}\right\} \\
& \leq 0(1) \sum_{n=1}^{m}\left|\triangle_{n}\right|^{k} \\
& =0(1) \sum_{n=1}^{m} \beta_{n}^{k-1}\left|\triangle T_{n-1}\right|^{k} .
\end{aligned}
$$

Therefore

$$
\sum_{n=1}^{m} \alpha_{n}^{k-1}\left(\frac{p_{n}}{P_{n}}\right)^{k}\left|\frac{1}{R_{n-1}} \sum_{v=1}^{n-1} P_{v} q_{n-v-1} \frac{U_{v-1}}{u_{v}} \Delta \epsilon_{v} \Delta T_{v-1}\right|^{k}=0(1) \sum_{n=1}^{m} \beta_{n}^{k-1}\left|\Delta T_{n-1}\right|^{k}
$$

Now, put $\Delta T_{n-1}=\left(\frac{p_{n}}{P_{n}}\right)\left(\frac{\alpha_{n}}{\beta_{n}}\right)^{1-1 / k}$, we obtain

$$
\sum_{n=1}^{m} \alpha_{n}^{k-1}\left(\frac{p_{n}}{P_{n}}\right)^{k}\left|\frac{1}{R_{n-1}} \sum_{v=1}^{n-1} p_{v} q_{n-v-1}\left\{\left(\frac{U_{v-1}}{u_{v}}\right)\left(\frac{\alpha_{v}}{\beta_{v}}\right)^{1-1 / k} \Delta \epsilon_{v}\right\}\right|^{k}=0(1) \sum_{n=1}^{m} \alpha_{n}^{k-1}\left(\frac{p_{n}}{P_{n}}\right)^{k}
$$

This should imply

$$
\frac{1}{R_{n-1}} \sum_{v=1}^{n-1} p_{v} q_{n-v-1}\left\{\left(\frac{U_{v-1}}{u_{v}}\right)\left(\frac{\alpha_{v}}{\beta_{v}}\right)^{1-1 / k} \Delta \epsilon_{v}\right\}=0(1)
$$

But $\sum_{v=1}^{n-1} p_{v} q_{n-v-1}=R_{n-1}$, we get

$$
\left(\frac{U_{v-1}}{u_{v}}\right)\left(\frac{\alpha_{v}}{\beta_{v}}\right)^{1-1 / k} \Delta \epsilon_{v}=0(1)
$$

This completes the proof of the theorem.

REMARK. It is clear that

$$
\left|\bar{N}, p_{n}, P_{n} / p_{n}\right|_{k}=\left|\bar{N}, p_{n}\right|_{k}, \quad\left|\bar{N}, p_{n}, n\right|_{k}=\left|R, p_{n}\right|_{k}, \quad|\bar{N}, 1, n|_{k}=|C, 1|_{k},
$$

and from our definition we may deduce that

$$
q_{n}=1 \Rightarrow\left|N, \quad P_{n}, \phi_{n}\right|_{k}=\left|\bar{N}, p_{n}, \phi_{n}\right|_{k}
$$

which implies

$$
\left|N, P_{n}, P_{n} / p_{n}\right|_{k}=\left|\bar{N}, p_{n}\right|_{k}
$$

and

$$
\left|N, P_{n}, n\right|_{k}=\left|R, p_{n}\right|_{k} \cdot p_{n}=1 \Rightarrow\left|N, Q_{n}, n\right|_{k}=\left|N, q_{n}\right|_{k} .
$$

\section{APPLICATIONS}

COROLLARY 1. Let $P_{n} u_{n}=0\left(p_{n} U_{n}\right)$. Then the necessary and sufficient conditions that $\sum a_{n} \epsilon_{n}$ be summable $\left|\bar{N}, p_{n}\right|_{k}$ whenever $\sum a_{n}$ is summable $\left|\bar{N}, u_{n}\right|_{k}, \quad k \geq 1$, are 


$$
\epsilon_{n}=0\left\{\left(\frac{u_{n} P_{n}}{U_{n} p_{n}}\right)^{1 / k}\right\}, \quad \Delta \epsilon_{n}=0\left\{\left(\frac{u_{n}}{U_{n-1}}\right)\left(\frac{p_{n}}{P_{n}}\right)^{1-1 / k}\right\} .
$$

PROOF. Follows from the theorem by putting $q_{n}=1, \alpha_{n}=P_{n} / p_{n}$, and $\beta_{n}=U_{n} / u_{n}$

COROLLARY 2 (Bor and Thorpe [3]) Let $P_{n} u_{n}=0\left(p_{n} U_{n}\right)$ and $p_{n} U_{n}=0\left(P_{n} u_{n}\right)$ Then $\sum a_{n}$ is summable $\left|\bar{N}, p_{n}\right|_{k}$ iff it is summable $\left|\bar{N}, u_{n}\right|_{k}, \quad k \geq 1$

PROOF. Follows from Corollary 1 by putting $\epsilon_{n}=1$

COROLLARY 3. Let $Q_{n-1} u_{n}=0\left(U_{n}\right)$ Then the necessary and sufficient conditions that $\sum a_{n} \epsilon_{n}$ be summable $\left|N, q_{n}\right|_{k}$ whenever $\sum a_{n}$ is summable $\left|\bar{N}, u_{n}\right|_{k}, k \geq 1$, are

$$
\epsilon_{n}=0\left\{\left(\frac{Q_{n-1}}{n}\right)\left(\frac{n u_{n}}{U_{n}}\right)^{1 / k}\right\}, \quad \Delta \epsilon_{n}=0\left\{\left(\frac{u_{n}}{U_{n-1}}\right)\left(\frac{U_{n}}{n u_{n}}\right)^{1-1 / k}\right\} .
$$

PROOF. Follows from the theorem by putting $p_{n}=1, \alpha_{n}=n, \beta_{n}=U_{n} / u_{n}$ and making use of Lemma 2.

COROLLARY 4. Let $Q_{n-1} u_{n}=0\left(U_{n}\right)$. Then a necessary and sufficient condition that $\sum a_{n}$ be summable $\left|N, q_{n}\right|_{k}$ whenever it is summable $\left|\bar{N}, u_{n}\right|_{k}, \quad k \geq 1$, is

$$
n^{k-1} U_{n}=0\left(Q_{n-1}^{k} u_{n}\right) \text {. }
$$

PROOF. Follows from Corollary 3 by putting $\epsilon_{n}=1$.

COROLLARY 5. Let $\left\{n^{1-1 / k} p_{n} / P_{n} P_{n-1}\right\}$ nonincreasing, $P_{n} u_{n}=0\left(p_{n} U_{n}\right)$, and

$$
\sum_{n=v}^{\infty} \frac{n^{k-1} p_{n}^{k}}{P_{n}^{k} P_{n-1}}=0\left(\frac{v^{k-1} p_{v}^{k-1}}{P_{v}^{k}}\right) \text {. }
$$

Then the necessary and sufficient conditions that $\sum a_{n} \epsilon_{n}$ be summable $\left|R, p_{n}\right|_{k}$ whenever $\sum a_{n}$ is summable $\left|\bar{N}, u_{n}\right|_{k}, \quad k \geq 1$, are

$$
\epsilon_{n}=0\left\{\left(\frac{P_{n}}{n p_{n}}\right)\left(\frac{n u_{n}}{U_{n}}\right)^{1 / k}\right\}, \quad \Delta \epsilon_{n}=0\left\{\left(\frac{u_{n}}{U_{n-1}}\right)\left(\frac{U_{n}}{n u_{n}}\right)^{1-1 / k}\right\} .
$$

PROOF. Follows from the theorem by putting $q_{n}=1, \alpha_{n}=n$ and $\beta_{n}=U_{n} / u_{n}$.

COROLLARY 6. Let $P_{n} u_{n}=0\left(p_{n} U_{n}\right)$. Then the necessary and sufficient conditions that $\sum a_{n} \epsilon_{n}$ be summable $\left|\bar{N}, p_{n}\right|_{k}$ whenever $\sum a_{n}$ is summable $\left|R, u_{n}\right|_{k}, k \geq 1$, are

$$
\epsilon_{n}=0\left\{\left(\frac{n u_{n}}{U_{n}}\right)\left(\frac{P_{n}}{n p_{n}}\right)^{1 / k}\right\}, \quad \Delta \epsilon_{n}=0\left\{\left(\frac{u_{n}}{U_{n-1}}\right)\left(\frac{n p_{n}}{P_{n}}\right)^{1-1 / k}\right\} .
$$

PROOF. Follows from the theorem by putting $q_{n}=1, \alpha_{n}=P_{n} / p_{n}$ and $\beta_{n}=n$

The following four results follows from Corollary 3 and they are generalizations for the results of [8].

COROLLARY 7. The necessary and sufficient conditions that $\sum a_{n} \epsilon_{n}$ are summable $|C, \alpha|_{k}$, $0 \leq \alpha \leq 1$, whenever $\sum a_{n}$ is summable $|C, 1|_{k}, \quad k \geq 1$, are

$$
\epsilon_{n}=0\left(n^{\alpha-1}\right), \quad \Delta \epsilon_{n}=0\left(n^{-1}\right) .
$$

PROOF. Follows by putting $q_{n}=A_{n}^{\alpha-1}, u_{n}=1$.

COROLLARY 8. The necessary and sufficient conditions that $\sum a_{n} \epsilon_{n}$ be summable $|N, 1 /(n+1)|_{k}$ whenever $\sum a_{n}$ is summable $|C, 1|_{k}, k \geq 1$, are

$$
\epsilon_{n}=0(\log n / n), \quad \Delta \epsilon_{n}=0\left(n^{-1}\right) .
$$


PROOF. Follows by putting $q_{n}=1 /(n+1), u_{n}=1$

COROLLARY 9. The necessary and sufficient conditions that $\sum a_{n} \epsilon_{n}$ be summable $|N, 1 /(n+1)|_{k}$ whenever $\sum a_{n}$ is summable $|R, \log n, 1|_{k}, \quad k \geq 1$, are

$$
\epsilon_{n}=0\left\{(\log n)^{1-1 / k} / n\right\}, \quad \quad \quad \epsilon_{n}=0\left\{1 / n(\log n)^{1 / k}\right\} .
$$

PROOF. Follows by putting $q_{n}=u_{n}=1 /(n+1)$.

COROLLARY 10. The necessary and sufficient conditions that $\sum a_{n} \epsilon_{n}$ be summable $|C, \alpha|_{k}$, $0 \leq \alpha \leq 1$, whenever $\sum a_{n}$ is summable $|R, \log n, 1|_{k}, \quad k \geq 1$, are

$$
\epsilon_{n}=0\left\{n^{\alpha-1} /(\log n)^{1 / k}\right\}, \quad \Delta \epsilon_{n}=0\left\{1 / n(\log n)^{1 / k}\right\} .
$$

PROOF. Follows by putting $q_{n}=A_{n}^{\alpha-1}, u_{n}=1 /(n+1)$.

Lastly it may be mentioned that many other results could be obtained either from the theorem or from its corollaries.

\section{REFERENCES}

[1] BOR, H., A note on two summability methods, Proc. Amer. Math. Soc. 98 (1986), 81-84

[2] BOR, H., On the relative strength of two absolute summability methods, Proc. Amer. Math. Soc. 113 (1991), 1009-1012.

[3] BOR, H. and THORPE, B., On some absolute summability methods, Analysis 7 (1987), 145-152.

[4] DAS, G., Tauberian theorems for absolute Nörlund summability, Proc. Lond. Math. Soc. 19 (1969), 357-384.

[5] SUlaIMAN, W. T., Notes on two summability methods, Pure Appl. Math. Sci. 31 (1990), 59-68

[6] SUlaimaN, W T., On some summability factors of infinite series, Proc. Amer. Math. Soc. 115 (1992), 313-317.

[7] SULAIMAN, W. T., Relations on some summability methods, Proc. Amer. Math. Soc. 118 (1993), 1139-1145.

[8] MAZHAR, S. M., On the absolute Nörlund summability factors of infinite series, Proc. Amer. Math. Soc. 32 (1972), 233-236 


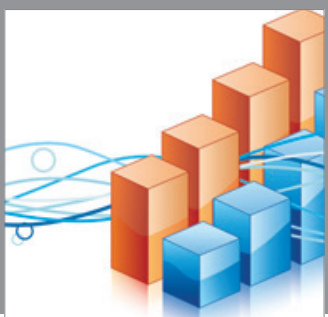

Advances in

Operations Research

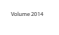

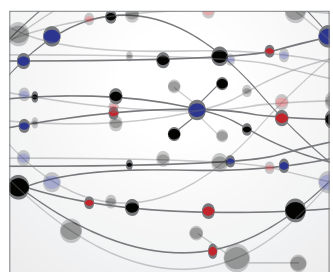

\section{The Scientific} World Journal
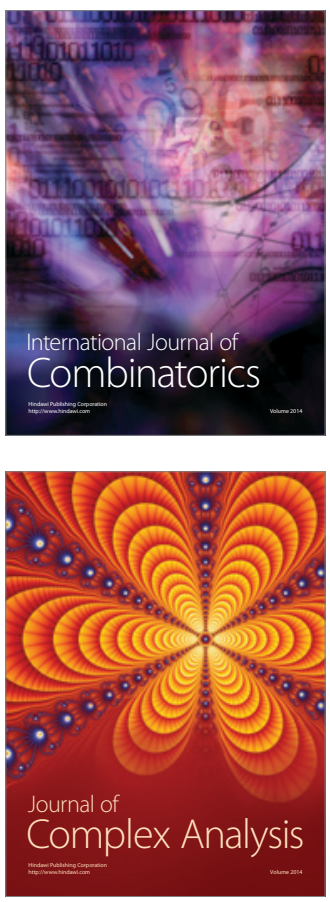

International Journal of

Mathematics and

Mathematical

Sciences
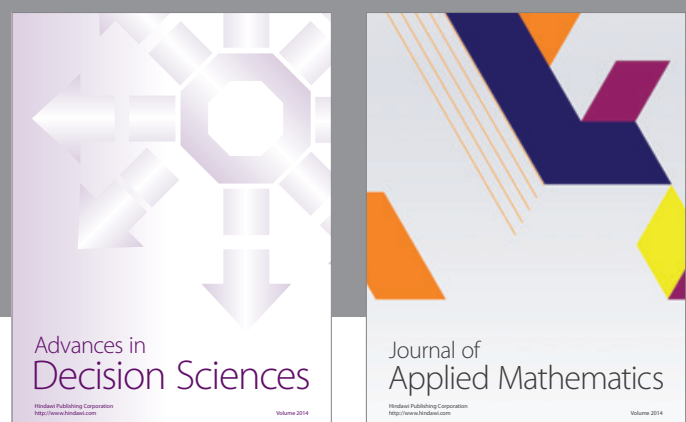

Journal of

Applied Mathematics
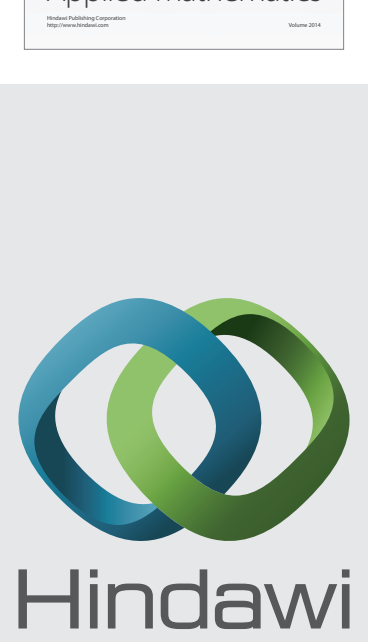

Submit your manuscripts at http://www.hindawi.com
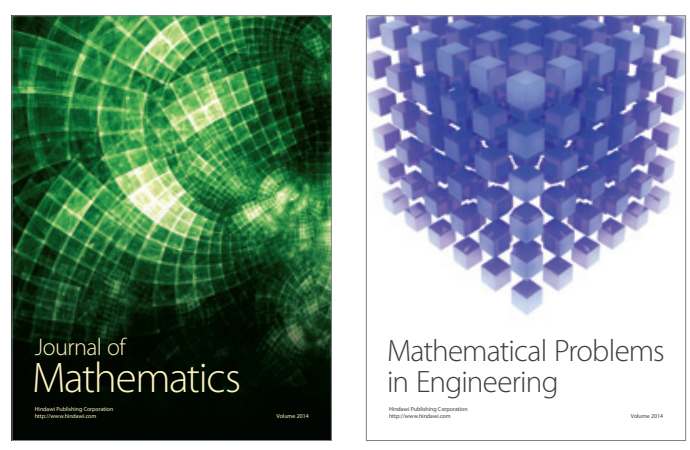

Mathematical Problems in Engineering
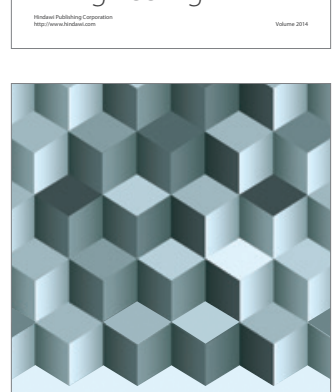

Journal of

Function Spaces
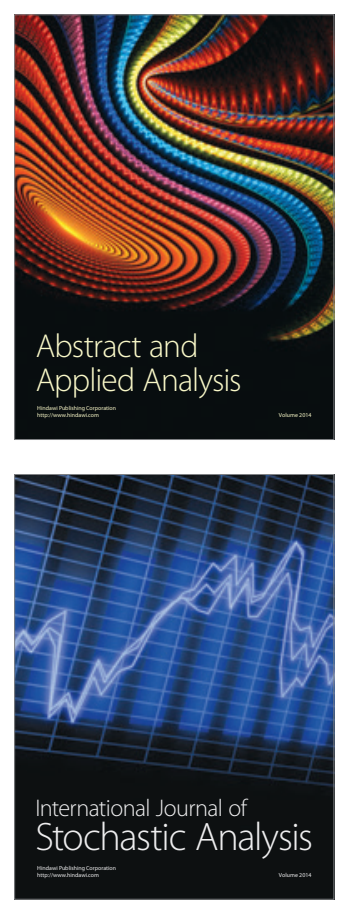

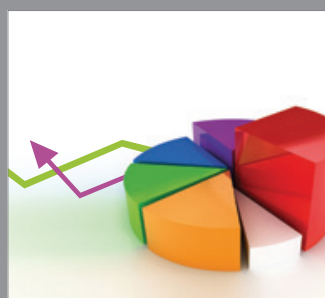

ournal of

Probability and Statistics

Promensencen
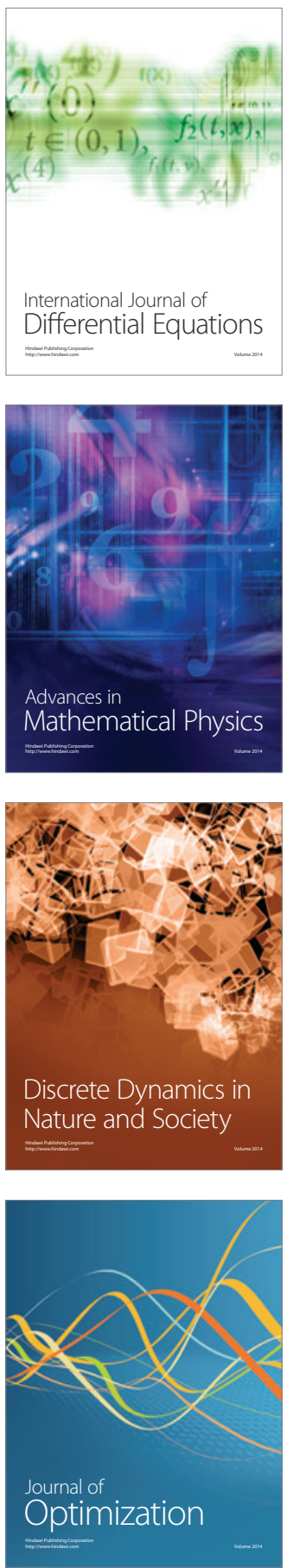